Loren D. Pitt

Nagoya Math. J.

Vol. 57 (1975), 199-228

\title{
HIDA-CRAMÉR MULTIPLICITY THEORY FOR MULTIPLE MARKOV PROCESSES AND GOURSAT REPRESENTATIONS
}

\author{
LOREN D. PITT
}

\section{Introduction.}

This work grew out of an attempt to prove the false result that an $n$-ple Markov process in the sense of Hida (1960) or Lévy (1956a) has multiplicity one. Instead we proved the representation theorem (Theorem III. 1.) that a centered Gaussian process $x(t)$ is $n$-ple Markov iff it can be written in the form

$$
x(t)=\sum_{1}^{n} e_{i}(t) a_{i}(t)
$$

where $A(t)=\left\{a_{i}(t)\right\}_{i=1, \ldots, n}$ is a Gaussian martingale with

$$
\operatorname{sp}\{x(s): s \leq t\} \equiv \operatorname{sp}\left\{a_{i}(s): s \leq t \text { and } 1 \leq i \leq n\right\}
$$

and $A(t)$ and $\left\{e_{i}(t)\right\}$ satisfy some non-degeneracy condition. We also show (Corollary IV. 13.) that for any Gaussian martingale $A(t)$ with simple left innovation spectrum, continuous $e_{i}(t)$ may be found so that the process $x(t)$ given in (I.1) will satisfy (I.2).

Together these results show that the only restrictions of the possible spectral type of an $n$-ple Markov process is that it has multiplicity $M \leq n$. These constitute our main results on $n$-ple Markov processes and the remainder of the paper is devoted to studying the implications that a process $x(t)$ admits a "Goursat" representation of the form (I.1).

Section II contains preliminaries on multiplicity theory and Gaussian martingales. In Section III, we prove the basic Theorem III. 1 mentioned above and derive analogous results for the covariance functions. Section IV developes the basic theory of Goursat representations. IV. 3

Received August 27, 1974.

Research supported in part by Army Research Office, Grant DA-ARO-D-31-124-71G182. AMS 1970 subject classifications. Primary 60G15 and 60G25. 
gives criteria which imply multiplicity one and is closely related to the work of Lévy in (1951) and (1956b). IV. 4 is devoted to deriving a condition on $e(t)$ which implies the equality (1.2). As a consequence we obtain Corollary IV. 13 mentioned earlier. The final Section V discusses the implications of smoothness properties of $x(t)$ on the multiplicity of $A(t)$. Roughly stated we show that if $x(t)$ has $k$ continuous derivatives then $A(t)$ has multiplicity no greater than $n-k$.

It is a pleasure to acknowledge the influence of the recent papers by V. Mandrekar (1974) and M. Hitsuda (1973). I received copies of these during the preparation of the present manuscript and because of insights obtained, the present work was greatly enhanced. Specifically Mandrekar proved independently the basic Theorem III. 1, under a very mild technical condition. The terminology "Goursat representation" is Mandrekar's as is part of Lemma IV. 1. Hitsuda's main results are special cases of the present Corollary IV. 9 and Theorem IV. 12, which when combined with our earlier versions lead directly to the present general formulations.

\section{Preliminaries.}

\section{1. Notations and multiplicity.}

We will write $x(t)$ for a centered real Gaussian process with $0<t$ $<\infty$, and $A(t)=\left\{a_{i}(t)\right\}_{i=1, \ldots, n}$ will denote a centered $n$-variate Gaussian process which we view as a column vector. We adopt standard matrix notation and denote transposes with *'s. Thus, if $e(t)=\left\{e_{i}(t)\right\}_{i=1, \ldots, n}$ and $f(t)=\left\{f_{i}(t)\right\}_{i=1, \ldots, n}$ are vector functions while $G(t)=\left(g_{i j}(t)\right)$ is an $n \times n$ matrix function we write $e^{*}(t) G(t) f(t)=\sum e_{i}(t) g_{i j}(t) f_{i}(t)$, and $e^{*}(t) A(t)=\sum e_{i}(t) a_{i}(t)$ will denote the inner product.

We consider $x(t)$ as a curve in the Hilbert space $L^{2}(\Omega)$ of square integrable random variables with inner product $\left\langle x_{1}, x_{2}\right\rangle=E x_{1} x_{2}$ and norm $\|x\|^{2}=E x^{2}$. Similarly $A(t)$ is considered as a vector curve with component functions $a_{i}(t) \in L^{2}(\Omega)$. Corresponding to $A(t)$ we denote the closed subspaces of $L^{2}(\Omega)$ :

$$
\begin{aligned}
& \mathscr{H}(A, t)=\operatorname{sp}\left\{a_{i}(s) ; s \leq t, 1 \leq i \leq n\right\} . \\
& \mathscr{H}(A)=\bigvee_{t} \mathscr{H}(A, t), \quad \mathscr{H}(A, t+)=\bigcap_{s>t} \mathscr{H}(A, s) .
\end{aligned}
$$

If $\mathscr{H}(A, 0+)=\{0\}$ we call $A(t)$ regular. $\mathscr{E}_{t}(A)$ denotes the orthogonal projection onto $\mathscr{H}(A, t)$. Similarly we define $\mathscr{H}(x, t), \mathscr{H}(x)$, and $\mathscr{E}_{t}(x)$.

The operator function $\left\{\mathscr{E}_{t}(A), 0 \leq t<\infty\right\}$ is monotonically non- 
decreasing, but in general $\mathscr{E}_{t}(A)$ is neither left nor right continuous. Because of this $\mathscr{E}_{t}(A)$ fails to be a spectral resolution of the identity on $\mathscr{H}(A)$ with the usual definition, but in his fundamental paper [1] Cramér has shown how the usual multiplicity theory for a spectral resolution extends to the present case. The only feature of this extension that we wish to emphasize is that innovation (discrete) spectrum splits into left and right parts and we may speak of left and right innovation multiplicities. Cramér shows that the left innovation multiplicity of $A(t)=$ $\left\{a_{i}(t)\right\} i=1, \cdots, n$ is bounded by $n$ and if $A(t)$ is left continuous there is no left innovation spectrum. We also mention that we will deviate slightly from the now standard usage and we will refer to the Hida-Cramér multiplicity $M$ of $\{A(t)\}$ as the maximum of the left innovation multiplicity $L$, the right innovation multiplicity $R$ and the continuous multiplicity $C$ rather than the usual $\max \{L+R, C\}$.

\section{2. Gaussian martingales.}

Let $A(t)$ be a centered $n$-variate Gaussian process and write $\mathscr{E}_{t}=\mathscr{E}_{t}(A)$. $A(t)$ is a martingale provided

$$
\mathscr{E}_{s} A(t)=A(s) \quad \text { for } s \leq t
$$

or equivalently, if

$$
\begin{gathered}
\left\langle a_{i}(r),\left[a_{j}(t)-a_{j}(s)\right]\right\rangle=0 \\
\text { for } r \leq s \leq t \text { and } 1 \leq i, j \leq n .
\end{gathered}
$$

With the martingale $A(t)$ we associate the structure matrix

$$
G(t)=\left(g_{i j}(t)\right)=\left(\left\langle a_{i}(t), a_{i}(t)\right\rangle\right) .
$$

Then

$$
\left\langle a_{i}(s), a_{j}(t)\right\rangle=g_{i j}(\min (s, t))
$$

and $G(t)$ determines the covariance structure of the process $A(t)$. One easily checks that for $t \geq s$

$$
G(t)-G(s)=E[A(t)-A(s)][A(t)-A(s)]^{*},
$$

and thus $G(t)$ is a non-decreasing function of non-negative matrices. Conversely any such function $G(t)$ is the structure matrix of some martingale $A(t)$.

$A(t)$ is regular iff $G(0+)=0$ and more generally, $\operatorname{dim} \mathscr{H}(A, 0+)=$ rank $G(0+)$. $A(t)$ will be called non-singular if the matrix $G(t)$ is non- 
singular for each $t$. From (II.4) we observe that $A(t)$ has left and right hand $L^{2}$ limits at each $t$ and that the points of left (right) discontinuity for $A(t)$ coincide with the points of left (right) discontinuity for $G(t)$.

The multiplicity theory for Gaussian matingales and the theory of stochastic "Wiener" integrals is well known and is presented in various degrees of completeness in a number of sources, e.g. Mandrekar (1968), Rosenberg (1964) and Rozanov (1958). Unfortunately our requirements do not allow the usual normalization of assuming $G(t)$ is right continuous. In the remainder of this section we briefly describe the necessary modifications in the existing theory.

For each point $t$ at which $G(t+) \neq G(t)$ we adjoin an ideal point $t+$ to the interval $[0, \infty)$ which is greater than $t$ but with $t+<s$ for each real $s>t$. The resulting set $\mathrm{I}$ is naturally ordered and the structure matrix $G(t)$ induces a non-negative matrix valued measure $G(d t)$ on the order $\sigma$-field $\mathscr{B}$ of $I$. Similarly the martingale $A(t)$ induces an $n$ variate Gaussian random measure $A(d t)$ on $\mathscr{B}$ with covariance measure $G(d t)$, i.e.

$$
E A\left(J_{1}\right) A^{*}\left(J_{2}\right)=G\left(J_{1} \cap J_{2}\right) \quad \text { for } J_{1}, J_{2} \in \mathscr{B} .
$$

We will write $L^{2}\{G\}$ for the $L^{2}$ space of vector functions $c(t)$ on $I$ with norm

$$
\|c\|^{2}=\int_{I} c^{*}(s) G(d s) c(s)<\infty
$$

For each $t \in I$ we write $L^{2}\{t, G\}$ for that subspace of $L^{2}\{G\}$ consisting of all functions supported on the interval $[0, t]$. With this notation the chain of spaces $\{\mathscr{H}(A, t), t \in I\}$ is naturally isomorphic to the chain $\left\{L^{2}\{t, G\} ; t \in I\right\}$, and the isomorphism is provided by the stochastic (Wiener) integral

$$
c(s) \rightarrow W(t)=\int^{t} c(s) A(d s),
$$

which gives a linear isometry from $L^{2}\{t, G\}$ onto $\mathscr{H}(A, t)$. The notation $\int^{t}$ will always be used for $\int_{[0, t]}$.

The multiplicity theory for $\{A(t)\}$ is easily established. Write $\mu(d t)$ for the trace measure of $G, \mu(d t)=\operatorname{tr} G(d t)$, and let $G^{\prime}(t)$ be the matrix density of $G(d t)$ with respect to $\mu . G^{\prime}(t)$ is a measurable matrix valued 
function and can be diagonalized with a measurable orthogonal matrix valued function $O(t)$, (see e.g. [2], p. 1341),

$$
O(t) G^{\prime}(t) O^{*}(t)=\operatorname{diag}\left(\gamma_{1}(t), \cdots, \gamma_{n}(t)\right)=\Gamma(t)
$$

with $\gamma_{1} \geq \gamma_{2} \geq \cdots \geq \gamma_{n} \geq 0$. Setting $\mu_{i}(d t)=\gamma_{i}(t) \mu(d t)$, we observe that

$$
\begin{aligned}
\int^{t} c^{*}(s) G(d s) c(s) & =\int^{t} e^{*}(s) \Gamma(s) e(s) \mu(d s) \\
& =\sum_{i=1}^{n} \int^{t} e_{i}^{2}(s) \mu_{i}(d s),
\end{aligned}
$$

where $e(t)=O(t) c(t)$. This shows that $c(s) \rightarrow O(t) c(s)$ determines a linear isometry

$$
L^{2}\{t, G\} \cong \sum_{i=1}^{n} L^{2}\left\{t, \mu_{i}\right\}
$$

and at the same time, shows that the multiplicity function for the resolution $\left\{\mathscr{E}_{t}\right\}$ is given by the rank function $r(t)=\operatorname{rank} G^{\prime}(t)=\operatorname{rank} \Gamma(t)$. Moreover, the multiplicity of an $n$-variate martingale $A(t)$ is no greater than $n$. The left innovation spectrum consists of those real $t$ for which $G(t)-G(t-) \neq 0$ with multiplicities $r(t)=\operatorname{rank}(G(t)-G(t-))$; similarly for the right innovation spectrum. When $G^{\prime}(t)$ has rank $k$ a.e. [ $\mu$ ] we say $A(t)$ has uniform multiplicity $k$ and when $k=1$ simply that $A(t)$ has multiplicity one or simple spectrum. When $\mu(t)$ is equivalent to Lebesgue measure we speak of Lebesgue spectrum.

\section{Multiple Markov processes.}

Let $x(t)$ be a univariate process and write $\mathscr{E}_{t}$ for $\mathscr{E}_{t}(x)$.

Definition III. 1. A process $x(t)$ will be called $n$-ple Markov if for all $a \leq b$, the set $\left\{\mathscr{E}_{a} x(t): t \geq b\right\}$ contains exactly $n$ linearly independent elements.

This definition differs from that of Hida (1960) where it is assumed that $\left\{\mathscr{E}_{a} x\left(t_{i}\right): i=1, \cdots, N\right\}$ contains exactly $n$ linearly independent elements whenever $a \leq t_{1}<\cdots<t_{N}$ are distinct and $N \geq n$.

Following Mandrekar (1974) we will say that a process $x(t)$ admits a Goursat representation of rank $n$ if there exists an $n$-variate martingale $A(t)$ and a (non-random) vector valued function $e(t)$ with

$$
X(t)=e^{*}(t) A(t)=\sum e_{i}(t) a_{i}(t) .
$$


If the martingale $A(t)$ is non-singular and the function $e(t)$ satisfies the condition:

For each $b$ there exist $t_{n}>t_{n_{-1}}>\ldots>t_{1} \geq b$ for which the matrix $\left(e_{i}\left(t_{j}\right)\right)$ is non-singular,

we say that (III.2) is a non-singular Goursat representation. If

$$
\mathscr{H}(x, t)=\mathscr{H}(A, t),
$$

we call representation (III.2) proper. Note that $\mathscr{H}(x, t) \subset \mathscr{H}(A, t)$ for any Goursat representation.

THEOREM III.1. $X(t)$ is n-ple Markov iff it admits a proper nonsingular Goursat representation of rank $n$.

Proof. Suppose $x(t)=e^{*}(t) A(t)$ is a proper Goursat representation of rank $n$. Then for $a \leq t, \mathscr{E}_{a} x(t)=e^{*}(t) A(a)$, and the set $\left\{\mathscr{E}_{a} x(t): t \geq b\right\}$ $=\left\{e^{*}(t) A(a): t \geq b\right\}$ certainly contains no more than $n$ independent elements. It will contain $n$ independent elements iff $A(t)$ is non-singular and there are times $t_{n}>\ldots>t_{1} \geq b$ for which $\left\{e\left(t_{i}\right): 1 \leq i \leq n\right\}$ are independent. This shows the sufficiency of the conditions of the theorem and also shows that to prove necessity one only needs establish the existence of a proper Goursat representation $x(t)=e^{*}(t) A(t)$.

Assuming $x(t)$ is $n$-ple Markov we now construct $A(t)$. We note that for $t \leq T$ the operator $\mathscr{E}_{t}$ induces a one-one invertable map from $\operatorname{sp}\left\{\mathscr{E}_{T} x(s): s \geq T\right\}$ onto $\operatorname{sp}\left\{\mathscr{E}_{t} x(s): s \geq t\right\}$. This is true because they both have dimension $n$, and the image $\operatorname{sp}\left\{\mathscr{E}_{t} \mathscr{E}_{T} x(s): s \geq T\right\}=\operatorname{sp}\left\{\mathscr{E}_{t} x(s): s \geq T\right\}$ also has dimension $n$. Thus we may fix a time $T_{0}$ and a basis $\left\{a_{i}\left(T_{0}\right)\right\}$ for $\operatorname{sp}\left\{\mathscr{E}_{T_{0}} x(s): s \geq T_{0}\right\}$ and then define the $a_{i}(t)$ by the formuli

$$
\begin{array}{r}
a_{i}(t)=\mathscr{E}_{t} a_{i}\left(T_{0}\right) \quad \text { if } \quad t \leq T_{0} \\
a_{i}\left(T_{0}\right)=\mathscr{E}_{T_{0}} a_{i}(t) \quad \text { and } \\
a_{i}(t) \in \operatorname{sp}\left\{\mathscr{E}_{t} x(s): s \geq t\right\} \quad \text { if } t>T_{0} .
\end{array}
$$

For the process $A(t)$ thus defined one has $\mathscr{H}(A, t) \subset \mathscr{H}(x, t)$. Because $\mathscr{E}_{t}: \operatorname{sp}\left\{\mathscr{E}_{T} x(s): s \geq T\right\} \rightarrow \operatorname{sp}\left\{\mathscr{E}_{t} x(s): s \geq t\right\}(T \geq t)$ is one-one the $\left\{a_{i}(t)\right\}$ form a basis of $\operatorname{sp}\left\{\mathscr{E}_{t} x(s): s \geq t\right\}$ and because $x(t) \in \operatorname{sp}\left\{\mathscr{E}_{t} x(s): s \geq t\right\}$ there will exist functions $e_{i}(t)$ for which 


$$
x(t)=\sum e_{i}(t) a_{i}(t) .
$$

This also shows that $\mathscr{H}(x, t)=\mathscr{H}(A, t)$.

It now remains to show that $A(t)$ is a martingale. For $s \leq t$ we show $\mathscr{E}_{s} A(t)=A(s)$ by checking the cases (i) $t \leq T_{0}$, (ii) $s \leq T_{0}<t$ and (iii) $T_{0}<s . \quad$ (i) $\mathscr{E}_{s} A(t)=\mathscr{E}_{s} \mathscr{E}_{t} A\left(T_{0}\right)=\mathscr{E}_{s} A\left(T_{0}\right)=A(s)$. (ii) $\mathscr{E}_{s} A(t)=\mathscr{E}_{s} \mathscr{E}_{T_{0}} A(t)$ $=\mathscr{E}_{s} A\left(T_{0}\right)=A(s)$. (iii) $\mathscr{E}_{T_{0}} \mathscr{E}_{s} A(t)=\mathscr{E}_{T_{0}} A(t)=A\left(T_{0}\right)$ and $\mathscr{E}_{T_{0}} A(s)=A\left(T_{0}\right)$. For each $j$, both $a_{j}(s)$ and $\mathscr{E}_{s} a_{j}(t)$ are in $\operatorname{sp}\left\{\mathscr{E}_{s} x(\tau): \tau \geq s\right\}$ and because $\mathscr{E}_{T_{0}}$ is one-one on $\operatorname{sp}\left\{\mathscr{E}_{s} x(\tau): \tau \geq s\right\}$ we see $a_{j}(s)=\mathscr{E}_{s} a_{j}(t)$. Thus $\mathscr{E}_{s} A(t)=$ $A(s)$. $A(t)$ is a martingale and the proof is complete.

Let $\rho(s, t)=E x(s) x(t)$ be the covariance function of an $n$-ple Markov process and let $G(t)$ be the structure matrix of the corresponding martingale $A(t)$ in Theorem III.1. Then

$$
\begin{aligned}
\rho(s, t) & =E e^{*}(s) A(s) e^{*}(t) A(t) \\
& =\sum e_{i}(s) E a_{i}(s) a_{j}(t) e_{j}(t) \\
& =\sum e_{i}(s) g_{i j}(\min (s, t)) e_{j}(t) .
\end{aligned}
$$

Setting $f(s)=G(s) e(s)$, we have

$$
\rho(s, t)=\sum_{i=1}^{n} f_{i}(s) e_{i}(t)=f^{*}(s) e(t), \quad s \leq t .
$$

The next proposition is elementary and we leave its verification to the reader.

Lemma III.2. A positive definite function $\rho(s, t)$ of the form

$$
\rho(s, t)=\sum_{i=1}^{n} f_{i}(\min (s, t)) e_{i}(\max (s, t))
$$

is the covariance function of an n-ple Markov process iff $f(s)$ and $e(t)$ satisfy.

For each $a>0$ there exist times $s_{1}<\ldots<s_{n} \leq a$ for which the matrix $\left(f_{i}\left(s_{j}\right)\right)$ is non-singular.

For each $b>0$ there exist times $t_{n}>\ldots>t_{1} \geq b$ for which the matrix $\left(e_{i}\left(t_{j}\right)\right)$ is non-singular.

The kernels $\rho$ given by (III.6) with $e(t)$ and $f(s)$ satisfying (III.7) have the following uniqueness property.

LEMMA III.3. Suppose $f(s)$ and $e(t)$ satisfy (III.7.a) and (III.7.b) 
and that $\tilde{f}(s)$ and $\tilde{e}(s)$ are another pair of $n$-dimensional vector valued functions satisfying

$$
f^{*}(s) e(t)=\tilde{f}^{*}(s) \tilde{e}(t), \quad s \leq t .
$$

Then there exists a unique non-singular matrix $L$ with

$$
\begin{aligned}
\tilde{e}(t) & =L e(t) \\
f(s) & =L^{*} \tilde{f}(s) .
\end{aligned}
$$

In particular, if $e(t) \equiv \tilde{e}(t)$ then $f(s) \equiv \tilde{f}(s)$.

Proof. Fix a time $T$ and choose times $t_{n}>\ldots>t_{1} \geq T$ for which $e\left(t_{1}\right), \cdots, e\left(t_{n}\right)$ are linearly independent. There exists a unique $L$ for which

$$
\operatorname{Le}\left(t_{i}\right)=\tilde{e}\left(t_{i}\right), \quad 1 \leq i \leq n .
$$

Then for $s \leq T$

$$
f^{*}(s) e\left(t_{i}\right)=\tilde{f}^{*}(s) L e\left(t_{i}\right), \quad 1 \leq i \leq n .
$$

Because the $e\left(t_{i}\right)$ are independent this shows that

$$
f(s)=L^{*} \tilde{f}(s) \quad \text { for } s \leq T,
$$

and condition (III.7a) implies that $L^{*}$ is invertible and that $\tilde{f}(s)$ satisfies condition (III.7a). From (III.9) we now have

$$
\tilde{f}(s) \tilde{e}(t)=f^{*}(s) e(t)=\tilde{f}^{*}(s) L e(t), \quad s \leq T \leq t
$$

and because $\tilde{f}(s)$ satisfies (III.7a)

$$
L e(t)=\tilde{e}(t), \quad t \geq T .
$$

Letting $T \downarrow 0$ while keeping $t_{1}, \cdots, t_{n}$ fixed shows that (III.10) holds for all $t$. Condition (III.7.a) and the identity (III.10) then gives $f(s)=L^{*} \tilde{f}(s)$ for all $s$ and thus completes the proof.

Proposition III.4. A kernel

$$
\rho(s, t)=f^{*}(\min (s, t)) e(\max (s, t)),
$$

where $f$ and $e$ satisfy (III.7), is positive definite iff there exists an increasing function $G(t)$ of non-negative matrices with

$$
f(t)=G(t) e(t)
$$


Proof. Suppose $\rho(s, t)$ is positive definite. By Lemma III.2 $\rho$ is the covariance function of an $n$-ple Markov process $x(t)$. By Theorem III.1 we can write $x(t)=\tilde{e}^{*}(t) A(t)$ where $A(t)$ is an $n$-variate martingale with structure matrix $H(t)$. Then for $s \leq t$

$$
\begin{aligned}
\rho(s, t) & =\tilde{e}^{*}(s) H(s) \tilde{e}(t) \\
& =\tilde{f}^{*}(s) \tilde{e}(t) \text { where } \tilde{f}(s)=H(s) \tilde{e}(s) .
\end{aligned}
$$

By Lemma III.3 there exists an invertible matrix $L$ with $\tilde{e}(t) \equiv L e(t)$ and $f(t)=L^{*} \tilde{f}(t)$. Thus $f(t)=L^{*} \tilde{f}(t)=L^{*} H(s) L e(s)=G(s) e(s)$ with $G(s)$ $=L^{*} H(s) L$, which is increasing and non-negative.

Conversely, if $f(t)$ satisfies (III.11) we may define $x(t)$ as $x(t)=$ $e^{*}(t) A(t)$ where $A(t)$ is a martingale with structure matrix $G(t)$. Then for $s \leq t$ we have

$$
\begin{aligned}
E x(s) x(t) & =e^{*}(s) G(s) e(t) \\
& =\rho(s, t),
\end{aligned}
$$

and $\rho(s, t)$ being a covariance function is positive definite. As a corollary to these arguments we state

COROLLARY III.5. A process $x(t)$ which admits a Goursat representation $x(t)=e^{*}(t) A(t)$ of rank $n$ is n-ple Markov iff the functions $e(t)$ and $f(t)=G(t) e(t)$ satisfy conditions (III.7.a) and (III.7.b). A kernel $\rho(s, t)$ is the covariance function of an n-ple Markov process iff it admits a representation of the form

$$
\rho(s, t)=e^{*}(s) G(s) e(t) \quad \text { for } s \leq t,
$$

where $e(t)$ satisfies (III.7.b), $G(s)$ is a non-decreasing function of positive definite matrices, and $f(t)=G(t) e(t)$ satisfies (III.7.a).

\section{Goursat representations.}

Whenever a Goursat representation

$$
x(t)=e^{*}(t) A(t)
$$

is known to be proper a number of problems are greatly simplified. For example, the least squares prediction of $x(t)$ given $\{x(\tau): \tau \leq s\}$ is

$$
\begin{aligned}
\mathscr{E}_{s} x(t) & =\mathscr{E}_{s}(A) e^{*}(t) A(t) \\
& =e^{*}(t) A(s) .
\end{aligned}
$$


The prediction error is

$$
\begin{aligned}
E\left|x(t)-\mathscr{E}_{s} x(t)\right|^{2} & =E\left|e^{*}(t)[A(t)-A(s)]\right|^{2} \\
& =e^{*}(t)[G(t)-G(s)] e(t) .
\end{aligned}
$$

The identity $\mathscr{H}(x, t) \equiv \mathscr{H}(A, t)$ shows that the Hida-Cramér multiplicity theories for the two processes $x(t)$ and $A(t)$ coincide and, as we shall see, leads directly to the Hida-Cramér canonical representation of $x(t)$. For these reasons it is desirable to have criteria for deciding if a Goursat representation is proper. In this section we present some basic theory for Goursat representations including canonical representations, criteria for properness, and multiplicity bounds for processes with Goursant representations.

\section{1. Existence of proper representations.}

LEMMA IV.1. Let $x(t)=e^{*}(t) A(t)$ be a Goursat representation and write $\mathscr{E}_{t}$ for $\mathscr{E}_{t}(x)$. Then:

$$
B(t) \equiv \mathscr{E}_{t} A(t) \text { is a martingale. }
$$

$$
x(t)=e^{*}(t) B(t) \text { and this representation is proper. }
$$

If in addition $x(t)$ is n-ple Markov then for any other proper Goursat representation

$$
x(t)=\tilde{e}^{*}(t) \tilde{B}(t)
$$

of $x(t)$ there exists a non-singular matrix $L$ with $\tilde{e}(t)=L e(t)$ and $B(t)=L^{*} \tilde{B}(t)$.

Proof. (IV.1): From the definition of $B(t), \mathscr{H}(B, t) \subset \mathscr{H}(x, t)$. Thus the identities

$$
\mathscr{E}_{s} B(t)=\mathscr{E}_{s} \mathscr{E}_{t} A(t)=\mathscr{E}_{s} A(t)=\mathscr{E}_{s} \mathscr{E}_{s}(A) A(t)=\mathscr{E}_{s} A(s)=B(s), \quad(s \leq t)
$$

show that $B(t)$ is a martingale.

(IV.2): That $x(t)=e^{*}(t) B(t)$ follows from

$$
x(t)=\mathscr{E}_{t} x(t)=\mathscr{E}_{t} e^{*}(t) A(t)=e^{*}(t) B(t) .
$$

This and the inclusion $\mathscr{H}(B, t) \subset \mathscr{H}(x, t)$ complete the proof. Item (IV.3) is due to Mandrekar (1974). The proof is an elementary modification of the proof of Lemma III.3, which we omit. 
Let $G(t)$ denote the structure matrix of $A(t)$ and set $f(t)=G(t) e(t)$. If $x(t)$ is $n$-ple Markov then, by Lemma III.3, any increasing solution $H(t)$ of

$$
E x(s) x(t)=e^{*}(s) H(s) e(t), \quad s \leq t
$$

must satisfy

$$
f(t)=H(t) e(t) .
$$

The covariance interpretation of (IV.3) is that equation (IV.4) has only one solution $H(t)$ corresponding to a proper representation; namely the structure matrix $H(t)$ of $B(t)=\mathscr{E}_{t} A(t)$. The covariance matrix of $A(t)$ $-B(t)$ is $G(t)-H(t)$. Thus $G(t)-H(t)$ is non-negative definite or $H(t)$ $\leq G(t)$, and this must hold for any other structure matrix solution $G(t)$ of (IV.4). We can thus state a corollary to Lemma IV.1.

COROLLARY IV.2. Let $x(t)$ be n-ple Markov and let

$$
x(t)=e^{*}(t) A(t)
$$

be a Goursat representation of rank $n$. Then (IV.5) is proper iff $G(t)$ is the minimal structure matrix solution of (IV.4).

\section{2. Multiplicity theory for Goursat representations.}

If $x(t)=e^{*}(t) A(t)$ is a proper Goursat representation the Hida-Cramér canonical representation for $x(t)$ is easily derived. This is done by diagonalizing the density $G^{\prime}(t)$ as in (II.5),

$$
O(t) G^{\prime}(t) O^{*}(t)=\Gamma(t)=\operatorname{diag} .\left(\gamma_{1}(t), \cdots, \gamma_{n}(t)\right),
$$

and setting

$$
B(t)=\int^{t} O(s) A(d s)
$$

Then $B(t)$ is a martingale with the diagonal structure matrix

$$
\begin{aligned}
E B(t) B^{*}(t) & =\int^{t} O(s) d G(s) O^{*}(s) \\
& =\int^{t} \Gamma(s) d \mu(s)
\end{aligned}
$$

Thus the processes $b_{j}(t)$ are independent martingales with $b_{j}(t)=0$ for $j>M$ where $M=$ ess. sup. $\operatorname{rank} G^{\prime}(s)$ is the multiplicity of $A(t)$. 
But (IV.6) is invertible with

$$
A(d s)=O^{*}(s) B(d s)
$$

and

$$
\begin{aligned}
x(t) & =e^{*}(t) A(t) \\
& =\int^{t} e^{*}(t) O^{*}(s) B(d s) \\
& =\sum_{j=1}^{M} \int^{t}\left(\sum_{i=1}^{n} e_{i}(t) O_{j i}(s)\right) b_{j}(d s) .
\end{aligned}
$$

Because $x(t)=e^{*}(t) A(t)$ is proper we have

$$
\mathscr{H}(x, t)=\mathscr{H}(A, t)=\mathscr{H}(B, t),
$$

and (IV.7) is seen to be the proper canonical Hida-Cramér representation of $x(t)$.

The Hida-Lévy criterion for canonical representations [Lévy (1956b) and Hida (1960)] also has a useful version in the present setting.

THEOREM IV.3. A Goursat representation $x(t)=e^{*}(t) A(t)$ is proper iff for each $T>0$ and $c \in L^{2}\{T, G\}$ the relation

$$
\begin{gathered}
0=e^{*}(t) \int^{t} G(d s) c(s) \quad \text { for all } t \leq T \text { implies } \\
G(d s) c(s) \equiv 0
\end{gathered}
$$

Proof. Because $\mathscr{H}(x, T) \subseteq \mathscr{H}(A, T)$ the representation will be proper iff the orthogonal complement of $\mathscr{H}(x, T)$ in $\mathscr{H}(A, T)$ is empty for each $T>0$. But each $x \in \mathscr{H}(A, T)$ has a representation as a stochastic integral

$$
x=\int^{T} c^{*}(s) d A(s), \quad c(s) \in L^{2}\{T, G\},
$$

and for $t \leq T$

$$
E x(t) x=e^{*}(t) \int^{t} G(d s) c(s)
$$

Thus $x$ is orthogonal to $\mathscr{H}(x, T)$ iff (IV.8) holds for each $t \leq T$ and the result follows.

\section{3. Multiplicity one conditions.}

In cases when $x(t)=c^{*}(t) A(t)$ has multiplicity one the general multi- 
plicity theory simplifies greatly and in certain cases Theorem IV.3 gives an effective technique for proving that a given Goursat representation is proper and in other cases for determining the proper representation. Our work in this section is closely related to that of Lévy in (1951) and (1956b) as well as Hitsuda (1973), but our techniques and results are quite different.

We begin by considering a Goursat representation

$$
x(t)=e^{*}(t) A(t)
$$

where the martingale $A(t)$ is regular (i.e. $G(0+)=0$ ) and the density $G^{\prime}=d G / d \mu$ of the structure matrix of $A(t)$ has rank 1 . Since $\mu(t)=$ $\operatorname{tr} G(t), \operatorname{tr} G^{\prime}(t)=1$ and $G^{\prime}(t)$ has the form

$$
G^{\prime}(t)=g(t) g^{*}(t)
$$

where $g(t)$ is a vector function with $|g(t)|=1$ a.e. $[\mu]$.

THEOREM IV.4. (i) The representation (IV.9) with $G^{\prime}(t)$ given in (IV.10) is proper iff for each $T>0, \alpha(t)=0$ is the only solution in $L^{2}\{T, \mu\}$ of

$$
0=\int^{t} e^{*}(t) g(s) \alpha(s) \mu(d s), \quad 0 \leq t \leq T
$$

(ii) A sufficient condition that (IV.9) is proper is that e(t) is absolutely continuous with respect to $\mu$ and that

$$
\left|\frac{d e}{d \mu}(t)\right|\left(e^{*}(t) g(t)\right)^{-1} \in L^{2}\{T, \mu\} \quad \text { for each } T>0 .
$$

Proof of (i). If $G^{\prime}(t)$ has the form (IV.10) then each function $\int^{t} G(d s) c(s)$ with $c(t) \in L^{2}\{T, G\}$ has a unique representation of the form

$$
\int^{t} G(d s) c(s)=\int^{t} g(s) \alpha(s) \mu(d s), \quad \alpha(t) \in L^{2}\{T, \mu\}
$$

In fact,

$$
\alpha(s)=g^{*}(s) c(s)
$$

and

$$
\int^{t} c^{*}(s) G(d s) c(s)=\int^{t}|\alpha(s)|^{2} \mu(d s)
$$


Thus

$$
e^{*}(t) \int^{t} G(d s) c(s)=\int^{t} e^{*}(t) g(s) \alpha(s) \mu(d s),
$$

and (i) follows directly from Theorem IV.3.

Proof of (ii). To see that (IV.12) is sufficient let $\alpha \in L^{2}\{T, \mu\}$ be such that

$$
0 \equiv \int^{t} e^{*}(t) g(s) \alpha(s) d \mu(s), \quad 0 \leq t \leq T .
$$

Differentiating with respect to $\mu$ gives

$$
0=e^{*}(t) g(t) \alpha(t)+\int^{t} \frac{d e^{*}}{d \mu}(t) g(s) \alpha(s) d \mu(s),
$$

or

$$
\alpha(t)=-\int^{t}\left(e^{*}(t) g(t)\right)^{-1} \frac{d e^{*}}{d \mu}(t) g(s) \alpha(s) d \mu(s) .
$$

Recalling that $|g(s)|=1$ a.e. [ $\mu$, condition (IV.12) shows that the Voltera kernel on the right side of (IV.14) is Hilbert-Schmidt. A Hilbert-Schmidt Voltera integral operator cannot have a non-zero eigenvalue and thus $\alpha \equiv 0$. Part (i) completes the proof.

Fortunately the quantity $e^{*}(t) g(t)$ has a direct interpretation in terms of the covariance function $\rho(s, t)=E x(s) x(t)$. To see this observe that

$$
\left[e^{*}(t) g(t)\right]^{2}=e^{*}(t) G^{\prime}(t) e(t)
$$

and that $r(t)=\rho(t, t)$ has the two representations $r(t)=e^{*}(t) G(t) e(t)$ and $r(t)=f^{*}(t) e(t)$. Differentiating each with respect to $\mu$ gives

$$
\frac{d r}{d \mu}(t)=e^{*}(t) G^{\prime}(t) e(t)+2 f^{*}(t) \frac{d e}{d \mu}(t)
$$

and

$$
\frac{d r}{d \mu}(t)=\frac{d f^{*}}{d \mu}(t) e(t)+f^{*}(t) \frac{d e}{d \mu}(t),
$$

so that

(IV.15)

$$
e^{*}(t) G^{\prime}(t) e(t)=\frac{d f^{*}}{d \mu}(t) e(t)-f^{*}(t) \frac{d e}{d \mu}(t)
$$


In case $\mu(t)$ is continuous we have also $e^{*}(t) G^{\prime}(t) e(t)=\lim _{h \downarrow 0} \frac{1}{\mu(t+h)-\mu(t)}\{\rho(t+h, t+h)+\rho(t, t)-2 \rho(t, t+h)\}$.

Finally, if we assume that $\mu(d t)$ is equivalent to Lebesgue measure we write $e^{\prime}(t)=d e / d t$ and set

$$
\sigma^{2}(t)=\lim _{h \downarrow 0} \frac{1}{h}\{\rho(t+h, t+h)-\rho(t, t)-2 \rho(t, t+h)\} .
$$

Then

$$
\sigma^{2}(t)=\left(\frac{d \mu}{d t}\right) e^{*}(t) G(t) e(t)
$$

and the dependence in condition (IV.12) on $\mu$ disappears giving

$$
\int^{T}\left|e^{\prime}(t)\right|^{2} \sigma^{-2}(t) d t<\infty
$$

The basic significance of this quantity $\sigma^{2}(t)$ was observed and exploited in a slightly different setting by Lévy in (1951) and (1956b).

We will now assume that $x(t)=e^{*}(t) A(t)$ is a representation for which $e(t)$ is absolutely continuous and $\sigma^{2}(t) \neq 0$ exists for almost all $t$. Assuming further that (IV.16) holds and setting $f(t)=G(t) e(t)$ we proceed to derive conditions under which the equation

$$
f(t)=H(t) e(t)
$$

can be solved with a structure matrix $H(t)$ that is absolutely continuous with respect to Lebesgue measure and for which $H^{\prime}(t)=d H / d t$ has rank 1. By the preceding comments and part (ii) of Theorem IV.4 this is equivalent to showing that $x(t)$ has simple Lebesgue spectrum.

If the desired matrix $H(t)$ exists then we may differentiate $f(t)=$ $H(t) e(t)$ and obtain

$$
f^{\prime}(t)=H(t) e^{\prime}(t)+H^{\prime}(t) e(t)
$$

Thus a necessary condition is that $f(t)$ is absolutely continuous. But assuming $H^{\prime}(t)$ has rank 1 , (IV.18) gives

$$
H^{\prime}(t)=\frac{\left(f^{\prime}(t)-H e^{\prime}(t)\right)\left(f^{\prime}(t)-H e^{\prime}(t)\right)^{*}}{e^{*}(t)\left(f^{\prime}(t)-H e^{\prime}(t)\right)}, \text { a.e. }[d t]
$$


The denominator is non-zero since

$$
\begin{aligned}
e^{*}(t) f^{\prime}(t)-e^{*}(t) H(t) e^{\prime}(t) & =f^{* \prime}(t) e(t)-f^{*}(t) e^{\prime}(t) \\
& =\sigma^{2}(t) \neq 0 \quad \text { a.e. }[d t] .
\end{aligned}
$$

Thus any absolutely continuous $H(t)$ with $\operatorname{rank} H^{\prime}(t)=1$ which satisfies (IV.17) must also satisfy the differential equation (IV.19). Conversely suppose that $H(t)$ is absolutely continuous on some interval $[a, b)$ with

$$
H(a) e(a)=f(a)
$$

and that (IV.19) holds on $[a, b)$. Then

$$
H^{\prime}(t) e(t)=f^{\prime}(t)-H e^{\prime}(t)
$$

or

$$
\frac{d}{d t}(H(t) e(t))=f^{\prime}(t)
$$

and since $H(a) e(a)=f(a)$ we see

$$
H(t) e(t)=f(t), \quad t \in[a, b) .
$$

Moreover $H(t)$ is increasing since by (IV.15)

$$
\begin{aligned}
H^{\prime}(t) & =\left(f^{\prime}(t)-H(t) e^{\prime}(t)\right)\left(f^{\prime}(t)-H(t) e^{\prime}(t)\right) * \sigma^{-2}(t) \\
& \geq 0 \quad \text { a.e. }
\end{aligned}
$$

These considerations allow us to draw the conclusion that $x(t)$ has multiplicity one if the equation (IV.19) has a local existence theorem. Precisely, we can prove

THEOREM IV.5. Suppose that

$$
\rho(s, t)=f^{*}(s) e(t) ; \quad s \leq t
$$

where $e(t)$ and $f(t)$ are absolutely continuous. Suppose also that $\sigma^{2}(t) \neq 0$ exists a.e. and that (IV.16) holds. A sufficient condition that $x(t)$ has simple Lebesgue spectrum in $(0, \infty)$ is that for each $T>0$ and each nonnegative matrix $H$ with

$$
H e(T)=f(T)
$$

the initial value problem 


$$
\begin{gathered}
H^{\prime}(t)=\frac{\left(f^{\prime}(t)-H(t) e^{\prime}(t)\right)\left(f^{\prime}(t)-H(t) e^{\prime}(t)\right)^{*}}{e^{*}(t)\left(f^{\prime}(t)-H(t) e^{\prime}(t)\right.} \\
H(T)=H
\end{gathered}
$$

has an absolutely continuous solution $H(t)$ on some interval $[T, T+\varepsilon)$.

Proof. We will show that for each $T>0$ an $\varepsilon>0$ can be found for which $x(t)$ has simple Lebesgue spectrum in the interval $[T, T+\varepsilon)$. Repeating this argument with $T$ replaced by $T+\varepsilon$ we can find a maximal interval $[T, S)$ with $T<S \leq+\infty$ in which $x(t)$ has simple Lebesgue spectrum. If $S<+\infty$ we can find another $\varepsilon>0$ so that $x(t)$ has simple Lebesgue spectrum in $[T, S) \cup[S, S+\varepsilon)=[T, S+\varepsilon)$, thus contradicting the assumption that $[T, S)$ was a maximal such interval. Thus $S$ must equal $+\infty$ and since $T>0$ was arbitrary $x(t)$ will have simple Lebesgue spectrum on $(0, \infty)$ plus possibly some right innovation spectrum at $t=0$ if $x(t)$ is not regular.

Proceeding we fix $T>0$ and let

$$
x(t)=e^{*}(t) B(t)
$$

be the proper Goursat representation of $x(t)$, whose existence is guaranteed by Lemma IV.1. Let $\dot{H}(t)$ denote the structure matrix of $B(t)$ and define $\tilde{H}(t)$ for $t \in[0, T+\varepsilon)$ by

$$
\tilde{H}(t)=H(t) \quad \text { if } \quad t \leq T,
$$

and for $T<t<T+\varepsilon$ let $\tilde{H}(t)$ denote the solution of (IV.20) with $\tilde{H}(T)$ $=H(T)$, whose existence we have assumed. Then $\tilde{H}(t)$ is a structure matrix solution of

$$
f(t)=\tilde{H}(t) e(t) ; \quad 0 \leq t<T+\varepsilon .
$$

Let $\tilde{B}(t)$ be a martingale defined on $(0, T+\varepsilon)$ with structure matrix $\tilde{H}(t)$, and set

$$
\tilde{x}(t)=e^{*}(t) \tilde{B}(t)
$$

Then for $s, t<T+\varepsilon$

$$
E \tilde{x}(s) \tilde{x}(t)=E x(s) x(t),
$$

and $x(t)$ and $\tilde{x}(t)$ have isomorphic multiplicity theories for $0<t<T+\varepsilon$. Because $x(t)=e^{*}(t) B(t)$ is proper and $H(t)=\tilde{H}(t)$ for $t \leq T$ we have 


$$
\mathscr{H}(\tilde{x}, t)=\mathscr{H}(\tilde{B}, t), \quad t \leq T
$$

The proof of Theorem IV.4 is now easily modified to show that

$$
\mathscr{H}(\tilde{x}, t)=\mathscr{H}(\tilde{B}, t) ; \quad t<T+\varepsilon .
$$

But $\tilde{H}(t)$ satisfies (IV.20) on $[T, T+\varepsilon$ ), and thus $\tilde{B}(t)$ has simple Lebesgue spectrum in $[T, T+\varepsilon)$. Hence $\tilde{x}(t)$ and $x(t)$ have simple Lebesgue spectrum in $[T, T+\varepsilon)$ and the proof is complete.

If $e^{\prime}(t)$ and $f^{\prime}(t)$ are continuous and $\sigma^{2}(t)>0$ for $t>0$ the classical existence theorem applies to the initial value problem (IV.20), and we have

CoRollaRy IV.6. If $e^{\prime}(t)$ and $f^{\prime}(t)$ are continuous and if $\sigma^{2}(t)>0$ for $t>0$ then $x(t)$ has simple Lebesgue spectrum.

It is not unreasonable to suppose that a local existence theorem for the initial value problem (IV.20) holds whenever $e(t)$ and $f(t)$ are absolutely continuous and $\left\{\left|e^{\prime}(t)\right|+f^{\prime}(t) \mid\right\} \sigma^{-1}(t)$ is locally square integrable. We have been unable to prove this but one can easily verify the following approximation theorem, whose proof we omit.

Proposition IV.7. The initial value problem (IV.20) has local solutions provided that

(IV.21) $\quad\left\{\left|e^{\prime}(t)\right|+\left|f^{\prime}(t)\right|\right\} \sigma^{-1}(t)$ is locally square integrable,

and

for each $T>0$ there exists an $\varepsilon>0$ and a sequence of positive definite functions

$\rho_{n}(s, t)=f_{n}^{*}(\min (s, t)) e_{n}(\max (s, t)) \quad T \leq s, t \leq T+\varepsilon$.

with continuously differentiable $e_{n}(t)$ and $f_{n}(t)$ for which $\sigma_{n}^{2}(t)>0$ on $[T, T+\varepsilon)$ and

$$
\lim _{n \rightarrow \infty} e_{n}(t)=e(t), \quad \lim _{n \rightarrow \infty} f_{n}(t)=f(t)
$$

and

$$
\lim _{n \rightarrow \infty} \int_{T}^{T+\varepsilon}\left\{\left|\frac{e_{n}^{\prime}(t)}{\sigma_{n}(t)}-\frac{e^{\prime}(t)}{\sigma(t)}\right|^{2}+\left|\frac{f_{n}^{\prime}(t)}{\sigma_{n}(t)}-\frac{f^{\prime}(t)}{\sigma(t)}\right|^{2}\right\} d t=0
$$

There are two elementary cases where this theorem is easily applied. 
THEOREM IV.8. Let $\rho(s, t)$ be the covariance function of a process with Goursat representation $x(t)=e^{*}(t) A(t)$. Set $f(t)=G(t) e(t)$ and suppose that $e(t)$ and $f(t)$ are both absolutely continuous and that $e^{\prime}(t)$ and $f^{\prime}(t)$ are both locally square integrable. If either

(IV.24) $\quad G^{\prime}(t)$ is continuous and $\sigma^{2}(t) \neq 0$ for all $t>0$ or $e(t) \neq 0$ for all $t, G^{\prime}(t)$ is locally square summable and the minimum eigenvalue of $G^{\prime}(t)$ is locally bounded below,

then the conditions of Proposition IV.7 are satisfied and $x(t)$ has simple Lebesgue spectrum on $(0, \infty)$.

Proof. If (IV.24) holds we simply take $e_{n}(t)$ to be a sequence of continuously differentiable functions with $\int_{T}^{T+s}\left|e_{n}^{\prime}(t)-e^{\prime}(t)\right|^{2} d t \rightarrow 0$. Setting $f_{n}(t)=G(t) e_{n}(t)$ we know that $\rho_{n}(s, t)=f_{n}^{*}(\min (s, t)) e_{n}(\max (s, t))$ is positive definite. Moreover, $f_{n}^{\prime}(t)=G^{\prime}(t) e_{n}(t)+G(t) e_{n}^{\prime}(t)$ is continuous and converges to $f^{\prime}(t)$ in the $L^{2}$ norm on $[T, T+\varepsilon)$. Upon noting that $\sigma_{n}^{2}(t)=e_{n}^{*}(t) G(t) e_{n}(t)$ converges uniformly to $\sigma^{2}(t)>0$ on $[T, T+\varepsilon)$, condition (IV.23) is obvious.

If (IV.25) holds we let $e_{n}$ be as above and let $G_{n}(t)$ be a continuously differentiable approximation of $G(t)$ with $\int_{T}^{T+\varepsilon}\left|G_{n}^{\prime}(t)-G(t)\right|^{2} d t \rightarrow 0$ and such that $G_{n}^{\prime}(t) \geq \delta I$ for all $t \in[T, T+\varepsilon]$ and some $\delta>0$. Setting $f_{n}(t)$ $=G_{n}(t) e_{n}(t)$ one can proceed as above.

CORollary IV.9 (See Hitsuda (1973)). Let $B(t)$ be a standard ndimensional Brownian motion and let $e(t)$ be absolutely continuous with

$$
\int^{T}\left|e^{\prime}(t)\right|^{2} d t<\infty, \quad \text { for } T<\infty
$$

and

$$
e(t) \neq 0 \quad \text { for all } t \text {. }
$$

Then

$$
\begin{aligned}
x(t) & =e^{*}(t) B(t) \\
& =e_{1}(t) b_{1}(t)+\cdots+e_{n}(t) b_{n}(t)
\end{aligned}
$$

has simple Lebesgue spectrum. 
EXAMPLE $(n=2)$. Let $n=2$ and let $x(t)=e^{*}(t) A(t)$ be a process with simple Lebesgue spectrum in $(0, \infty)$. Supposing that $e(t)$ is absolutely continuous and $\sigma^{2}(t)>0$ a.e. then we have seen that the problem of finding the structure matrix $H(t)$ of $B(t)$ for a proper Goursat representation $x(t)=e^{*}(t) B(t)$ corresponds to solving the equation

$$
H^{\prime}(t)=\frac{\left(f^{\prime}(t)-H(t) e^{\prime}(t)\right)\left(f^{\prime}(t)-H(t) e^{\prime}(t)\right)^{*}}{e^{*}(t)\left(f^{\prime}(t)-H(t) e^{\prime}(t)\right)}
$$

or, more simply, since $\sigma^{2}(t)=e^{*}(t)\left(f^{\prime}(t)-H(t) e^{\prime}(t)\right)$

$$
H^{\prime}(t)=\frac{\left(f^{\prime}(t)-H(t) e^{\prime}(t)\left(f^{\prime}(t)-H(t) e^{\prime}(t)\right)^{*}\right.}{\sigma^{2}(t)} .
$$

Equation (IV.26) is a quadratic equation in the three unknowns $h_{11}(t)$, $h_{22}(t)$ and $h_{12}(t)=h_{21}(t)$ but can be reduced to a simple Riccati equation as we will now show.

Denote the structure matrix of $A(t)$ by $G(t)$. Then we must have

$$
f(t)=G(t) e(t)=H(t) e(t) .
$$

Setting $J(t)=H(t)-G(t)$ we have

$$
J(t) e(t)=0 .
$$

Since $J(t)$ is symmetric we can write

$$
J(t)=\lambda(t)\left(\begin{array}{cc}
\left(e_{2}(t)\right)^{2} & -e_{1}(t) e_{2}(t) \\
-e_{1}(t) e_{2}(t) & \left(e_{1}(t)\right)^{2}
\end{array}\right)
$$

where $\lambda(t)$ is a scalar function. Moreover, $H^{\prime}(t)$ has rank one since $x(t)$ has simple Lebesgue spectrum in $(0, \infty)$ and hence

$$
\operatorname{det} H^{\prime}(t)=\operatorname{det}\left(G^{\prime}(t)+J^{\prime}(t)\right)=0 .
$$

A simple calculation now shows that (IV.27) is equivalent to the Riccati equation

$$
0=\lambda^{\prime}(t) a(t)+b(t)+c(t) \lambda(t)+d(t) \lambda^{2}(t)
$$

where

$$
\begin{aligned}
a(t) & =e_{2}^{2} g_{22}^{\prime}+e_{1}^{2} g_{11}^{\prime}+2 e_{1} e_{2} g_{12}^{\prime} \\
& =\sigma^{2}(t) \\
b(t) & =g_{11}^{\prime} g_{22}^{\prime}-\left(g_{12}^{\prime}\right)^{2}=\operatorname{det} G^{\prime}
\end{aligned}
$$




$$
\begin{aligned}
& c(t)=2\left[e_{2} e_{2}^{\prime} g_{22}^{\prime}+e_{1} e_{1}^{\prime} g_{11}^{\prime}+\left(e_{1} e_{2}^{\prime}+e_{1}^{\prime} e_{2}\right) g_{12}^{\prime}\right] \\
& d(t)=-\left(e_{1} e_{2}^{\prime}-e_{2} e_{1}^{\prime}\right)^{2} .
\end{aligned}
$$

As a simple example consider

$$
x(t)=\operatorname{Cos}(t) a_{1}(t)+\operatorname{Sin}(t) a_{2}(t)
$$

where $a_{1}(t)$ and $a_{2}(t)$ are independent Brownian motions. Then $e_{1}(t)=$ $\operatorname{Cos}(t), e_{2}(t)=\operatorname{Sin}(t), g_{11}^{\prime}=g_{22}^{\prime}=1$, and $g_{12}^{\prime}=0$. Equation (IV.28) simplifies to

$$
0=\lambda^{\prime}(t)+1-\lambda^{2}(t) .
$$

The initial condition $H(0)=0$ requires that $\lambda(0)=0$, and we find that $\lambda(t)=-\tanh (t)$ and

$$
H(t)=\left(\begin{array}{ll}
t & 0 \\
0 & t
\end{array}\right)-\tanh (t)\left(\begin{array}{cc}
\operatorname{Cos}^{2}(t) & -\operatorname{Sin}(t) \operatorname{Cos}(t) \\
-\operatorname{Sin}(t) \operatorname{Cos}(t) & \operatorname{Sin}^{2}(t)
\end{array}\right) .
$$

\section{4. Arbitraliness of $A(t)$ in proper Goursat representations.}

If $A(t)$ occurs in a proper Goursat representation

$$
x(t)=e^{*}(t) A(t),
$$

the equality $\mathscr{H}(x, t)=\mathscr{H}(A, t)$ shows that $A(t)$ has simple left innovation spectrum. We also know from the material in the last section that if $e(t)$ is too smooth with respect to $\mu(t)=\operatorname{tr} G(t)$ and $A(t)$ has multiplicity greater than one then (IV.29) cannot be expected to be proper. We now show that for any $A(t)$ with simple left innovation spectrum a continuous function $e(t)$ may be chosen for which (IV.29) is proper. Throughout this section the following assumptions will be in force.

$A(t)$ is an $n$-variate martingale whose left innovation spectrum

(IV.30) is simple. The trace of the structure matrix $G(t)$ of $A(t)$ will be denoted by $\mu(t)$.

(IV.31) $e(t)=\left(e_{1}(t), \cdots, e_{n}(t)\right)^{*}$ is $n-1$ times continuously differentiable.

$\tau(t)$ is a continuous positive function such that for each non empty interval $[a, b] \subset(0, \infty)$,

$$
\int_{0}^{a} \frac{(d \tau)^{2}}{d \mu} \equiv \sup \sum_{i} \frac{\left[\tau\left(t_{i+1}\right)-\tau\left(t_{i}\right)\right]^{2}}{\mu\left(t_{i+1}\right)-\mu\left(t_{i}\right)}=+\infty
$$

where this supremum is over all finite paritions of $[a, b]$. 
(IV.33) $\quad x(t)$ is the process with Goursat representation

$$
x(t)=e^{*}(\tau(t)) A(t) .
$$

Proposition IV.10. For $0<s<T$

$$
\left[e^{\prime}(\tau(T))\right]^{*} A(T-) \in \operatorname{sp}\{x(t): s<t \leq T\} .
$$

Proof. Fix $t \in(s, T)$ and for each partition $t_{0}=t \leq t_{1}<\cdots<t_{n}=T$ of $[t, T]$ satisfying $\tau\left(t_{i+1}\right) \neq \tau\left(t_{i}\right)$ we associate the sum

$$
x=\sum \lambda_{i} \frac{x\left(t_{i+1}\right)-x\left(t_{i}\right)}{\tau\left(t_{i+1}\right)-\tau\left(t_{i}\right)}
$$

where

$$
\lambda_{i}=\frac{\left[\tau\left(t_{i+1}\right)-\tau\left(t_{i}\right)\right]^{2}}{\mu\left(t_{i+1}\right)-\mu\left(t_{i}\right)}\left(\sum \frac{\left[\tau\left(t_{j+1}\right)-\tau\left(t_{j}\right)\right]^{2}}{\mu\left(t_{j+1}\right)-\mu\left(t_{j}\right)}\right)^{-1} .
$$

Then $\lambda_{i}>0$ and $\sum \lambda_{i}=1$.

Setting $\Delta_{i} \tau=\tau\left(t_{i+1}\right)-\tau\left(t_{i}\right)$ we write

$$
x=\sum \lambda_{i} e^{*}\left(\tau\left(t_{i+1}\right)\right) \frac{A\left(t_{i+1}\right)-A\left(t_{i}\right)}{\Delta_{i} \tau}+\sum \lambda_{i} \frac{\left[e\left(\tau\left(t_{i+1}\right)-e\left(\tau\left(t_{i}\right)\right)\right]^{*}\right.}{\Delta_{i} \tau} A\left(t_{i}\right) .
$$

By the mean value theorem there exist numbers $s_{i} \in\left(t_{i}, t_{i+1}\right)$ with $\left[e\left(\tau\left(t_{i+1}\right)-e\left(\tau\left(t_{i}\right)\right)\right]\left(\Delta_{i} \tau\right)^{-1}=e^{\prime}\left(\tau\left(s_{i}\right)\right)\right.$, and thus

$$
\begin{aligned}
\left\|x-\left[e^{\prime}(\tau(T))\right]^{*} A(T-)\right\| \leq & \left\|\sum \lambda_{i} e^{*}\left(\tau\left(t_{i+1}\right)\right)\left[\frac{A\left(t_{i+1}\right)-A\left(t_{i}\right)}{\Delta_{i} \tau}\right]\right\| \\
& +\left\|\sum \lambda_{i}\left[e^{\prime}\left(\tau\left(s_{i}\right)\right)\right]^{*} A\left(t_{i}\right)-\left[e^{\prime}(\tau(T))\right]^{*} A(T-)\right\| .
\end{aligned}
$$

Letting $\left.\omega(t)=\sup \left\{\|\left[e^{\prime}(\tau(s))\right]^{*} A(t)-\left[e^{\prime}(T)\right)\right]^{*} A(T-) \|: t \leq s \leq T\right\}$ we see $\omega(t) \downarrow 0$ as $t \uparrow T$ and that

$$
\begin{aligned}
\left\|\sum \lambda_{i}\left\{\left[e^{\prime}\left(\tau\left(s_{i}\right)\right)\right]^{*} A\left(t_{i}\right)-\left[e^{\prime}(\tau(T))\right]^{*} A(T-)\right\}\right\| & \leqq \sum \lambda_{i} \omega(t) \\
& =\omega(t) .
\end{aligned}
$$

Using the martingale property one obtains

$$
\begin{aligned}
& \left\|\sum \lambda_{i} e^{*}\left(\tau\left(t_{i+1}\right)\right)\left[\frac{A\left(t_{i+1}\right)-A\left(t_{i}\right)}{\Delta_{i} \tau}\right]\right\|^{2} \\
& \quad=\sum \lambda_{i}^{2}\left(\Delta_{i} \tau\right)^{-2} e^{*}\left(\tau\left(t_{i_{1}}\right)\right)\left[G\left(t_{i_{1}}\right)-G\left(t_{i}\right)\right] e\left(\tau\left(t_{i_{+1}}\right)\right) .
\end{aligned}
$$

Setting $M=\sup \{\|e(\sigma)\|: s \leq \sigma \leq T\}$ provides the estimate

$$
\begin{aligned}
0 & \leq e^{*}\left(\tau\left(t_{i+1}\right)\right)\left[G\left(t_{i+1}\right)-G\left(t_{i}\right)\right] e\left(\tau\left(t_{i+1}\right)\right) \\
& \leq M^{2}\left[\mu\left(t_{i+1}\right)-\mu\left(t_{i}\right)\right],
\end{aligned}
$$


which gives an upper bound for (IV.34) of

$$
M^{2} \sum \lambda_{i}^{2} \frac{\mu\left(t_{i_{1}}\right)-\mu\left(t_{i}\right)}{\left(\Delta_{i} \tau\right)^{2}}=M^{2}\left(\sum \frac{\left[\tau\left(t_{i+1}\right)-\tau\left(t_{i}\right)\right]^{2}}{\mu\left(t_{i+1}\right)-\mu\left(t_{i}\right)}\right)^{-1} .
$$

By (IV.32) this can be made less than $(T-t)$ with an appropriate choice: of the partition. For such a choice we have

$$
\left\|x-\left[e^{\prime}(\tau(t))\right]^{*} A(T-)\right\| \leq \sqrt{T-t}+\omega(t) .
$$

Noting again that $\omega(t) \downarrow 0$ as $t \uparrow T$ shows that $\left[e^{\prime}(\tau(T))\right]^{*} A(T-)$ is a limit point of the $x$ 's and hence is contained in $\operatorname{sp}\{x(\sigma): s<\sigma \leq T\}$.

The above argument applies equally well to the derivatives $e^{(k)}(t)$, $1 \leq k<n$, and we obtain the immediate

Corollary IV.11. For $0 \leq s<T$ and $0 \leq k<n$,

$$
\left[e^{(k)}(\tau(T))\right]^{*} A(T-) \in \operatorname{sp}\{x(t): s<t \leq T\} .
$$

If the functions $e_{1}(t), \cdots, e_{n}(t)$ satisfy the Wronski condition

$$
W\left(e_{1}(T), \cdots, e_{n}(T)\right) \neq 0
$$

then the vectors $e(T), e^{\prime}(T), \cdots, e^{(n-1)}(T)$ are linearly independent and Corollary IV.11 shows that

$$
a_{i}(T-) \in \operatorname{sp}\{x(t): T-\varepsilon \leq t \leq T\} \subseteq \mathscr{H}(x, T), \quad i=1, \cdots, n .
$$

If $A(t)$ were left continuous this would show that the representation. (IV.33) was proper. When $A(t)$ is not left continuous this requires a further condition.

THEOREM IV.12. Suppose the Wronski condition (IV.34) is satisfied for all $T$ (a dense set would suffice) and further that $e(t)$ is such that $[G(t)-G(t-)] e(\tau(t)) \neq 0$ whenever

$$
G(t)-G(t-) \neq 0
$$

Then for $T>\varepsilon>0$ and $1 \leq i \leq n$

$$
a_{i}(T) \in \operatorname{sp}\{x(t): T-\varepsilon<t \leq T\} .
$$

and the representation (IV.33) is proper.

Proof. We may assume $A(T)-A(T-) \neq 0$. Then by the preceding comments it suffices to show that 


$$
a_{i}(T)-a_{i}(T-) \in \operatorname{sp}\{x(t): T-\varepsilon \leq t \leq T\} .
$$

Moreover since $A(t)$ has simple left innovation spectrum, $A(T)-A(T-)$ has a degenerate normal distribution supported on the one dimensional range of $G(T)-G(T-)$. Thus the components $a_{i}(T)-a_{i}(T-)$ are scalar multiples of the variable

$$
e^{*}(\tau(T))[A(T)-A(T-)] .
$$

But

$$
e^{*}(\tau(T))[A(T)-A(T-)]=x(T)-x(T-) \in \operatorname{sp}\{x(t): T-\varepsilon \leq t \leq T\},
$$

and the proof is complete.

Comment. For any $A(t)$ with simple left innovation spectrum examples of functions $\tau(t)$ and $e(t)$ satisfying the conditions Theorem IV.12 exist. For example, let $\tau(t)$ be a strictly increasing continuous function that is singular with respect $\mu$ and choose $e(t)$ of the form

$$
e_{\alpha}(t)=(1, \exp (\alpha t), \cdots, \exp ((n-1) \alpha t)) .
$$

where $\alpha>0$ is such that

(IV.35) $\{G(t)-G(t-)\} e_{\alpha}(\tau(t)) \neq 0$ whenever $G(t)-G(t-) \neq 0$.

Such $\alpha$ 's must exist because for a fixed $t$ with $G(t) \neq G(t-)$ there are at most $(n-1)$ values of $\alpha$ with $\{G(t)-G(t-)\} e_{\alpha}(\tau(t))=0$, and there are at most countably many $t$ with $G(t) \neq G(t-)$. Thus (IV.35) holds for all but a coutable set of $\alpha>0$. For such an $\alpha$ the conditions of Theorem (IV.12) are satisfied and, moreover, if $t_{1}<\cdots<t_{n}$ then $\left(e_{i}\left(\tau\left(t_{j}\right)\right)\right)$ is nonsingular. By Theorem (III.1) and (IV.12) we have

COROLLARY IV.13. For any non-singular martingale $A(t)$ whose left innovation spectrum is simple there exists a continuous function e(t) so that the representation $x(t)=e^{*}(t) A(t)$ is proper and $x(t)$ is n-ple Markov.

Comment. A remarkable property of the processes described in Theorem IV.12 is that for all $t<T$,

$$
\operatorname{sp}\{x(s): t<s \leq T\}=\operatorname{sp}\left\{a_{i}(s): 1 \leq i \leq n, t<s \leq T\right\},
$$

or equivalently for each fixed $T$

$$
\bigcap_{t} \operatorname{sp}\{x(s): t<s \leq T\}=\bigcap_{t} \operatorname{sp}\left\{a_{i}(s): 1 \leq i \leq n, t<s \leq T\right\} .
$$

But for any Goursat representation 


$$
\bigcap_{t} \operatorname{sp}\{x(s): t<s \leq T\} \subseteq \bigcap \operatorname{sp}\left\{a_{i}(s): 1 \leq i \leq n, t<s \leq T\right\} .
$$

Thus the equality (IV.36) holds iff the dimensions of the two spaces in (IV.37) are equal. Assuming that $A(t)$ is left continuous and nonsingular

$$
\bigcap_{t} \operatorname{sp}\left\{a_{i}(s): 1 \leq i \leq n ; t<s \leq T\right\}=\operatorname{sp}\left\{a_{i}(T): 1 \leq i \leq n\right\}
$$

has dimension $n$. We thus have

Proposition IV.14. A Goursat representation $x(t)=e^{*}(t) A(t)$ where $A(t)$ is left continuous and non-singular is proper if for each $T$,

$$
\operatorname{dim} \bigcap_{t} \operatorname{sp}\{x(s): t<s \leq T\}=n .
$$

This is true in particular if $x(t)$ is $(n-1)$ times differentiable and for each $T,\left\{x(T), x^{\prime}(T), \cdots, x^{(n-1)}(T)\right\}$ are linearly independent. We show in section $\mathrm{V}$ that this condition implies that $x(t)$ has multiplicity one. In the opposite direction we now prove

Proposition IV.15. Suppose that $x(t)=e^{*}(t) A(t)$ is a proper Goursat representation of rank $n$ with uniform multiplicity $n$, (i.e. $G^{\prime}=d G / d \mu$ has rank $n$ a.e. $[\mu])$. Then for any $T>s>0$ with $\mu(T)>\mu(s+)$,

(IV.40) $\operatorname{sp}\{x(t): s<t \leq T\}=\operatorname{sp}\left\{a_{i}(t): 1 \leq i \leq n\right.$ and $\left.s<t \leq T\right\}$.

Proof. We begin with two simple observations. First, if $\mu(s+)<$ $\mu(T)$ then

$$
\operatorname{dim} \operatorname{sp}\{e(t): s<t \leq T\}=n .
$$

Second, if $x \in \operatorname{sp}\left\{a_{i}(t): 1 \leq i \leq n\right.$ and $\left.s<t \leq T\right\}$ then $x$ has a unique representation as a stochastic integral

$$
x=\int^{T} c^{*}(s) A(d s)
$$

with

$$
\begin{gathered}
c(t) \in L^{2}\{T, G\} \text { and } \\
c(t)=c(s+) \text { for } 0<t \leq s+.
\end{gathered}
$$

Thus if the equality (IV.40) does not hold, there exists a $c(t) \neq 0$ satisfying (IV.43) with 


$$
\begin{aligned}
0 & =E x(t) x \\
& =e^{*}(t) \int^{t} G(d s) c(s), \quad 0<t \leq T .
\end{aligned}
$$

We will show this is impossible, and to this end it suffices to show that (IV.44) implies $c(t) \equiv 0$ a.e. for $s<t \leq T$. This is true because (IV.43) and (IV.44) would give

$$
x=c^{*}(s+) A(s+)
$$

and

$$
0=E x(t) x=c^{*}(s+) G(s+) e(t), \quad s<t \leq T .
$$

By (IV.41) it follows that $G(s+) c(s+)=0$ and thus $E x^{2}=c^{*}(s+) G(s+)$ $\cdot c(s+)=0$, which contradicts the assumption that $c(t)$ is not identically zero.

To see that (IV.44) implies $c(t)=0$ for $s<t \leq T$ we define the function $\alpha(t)$ by

$$
\alpha(t)=0 \quad \text { for } 0<t \leq s+
$$

and

$$
\frac{d \alpha}{d \mu}(t)=\left\{1+\left|\left(G^{\prime}(t)\right)^{-1} \int^{t} G(d s) c(s)\right|\right\}^{-1}, \quad s+<t \leq T
$$

Setting

$$
c_{1}(t)=\left[G^{\prime}(t)\right]^{-1}\left\{\frac{d \alpha}{d \mu}(t) \int^{t} G(d s) c(s)\right\}+\alpha(t) c(t)
$$

we observe that $c_{1}(t) \in L^{2}\{T, G\}$ and that

$$
\int^{t} G(d s) c_{1}(s)=\alpha(t) \int^{t} G(d s) c(s), \quad 0 \leq t \leq T .
$$

Setting

$$
y=\int^{T} c_{1}^{*}(s) A(d s)
$$

gives

$$
E x(t) y=e^{*}(t) \int^{t} G(d s) c_{1}(s)
$$




$$
\begin{aligned}
& =\alpha(t) e^{*}(t) \int^{t} G(d s) c(s) \\
& =0, \quad 0<t \leq T .
\end{aligned}
$$

Because $x(t)=e^{*}(t) A(t)$ is assumed proper we may conclude that $y=0$. Thus $c_{1}(t) \equiv 0$ and by (IV.44), $c(t)=0$ for $s<t \leq T$. The proof is complete.

\section{Smoothness properties and multiplicity bounds.}

Smoothness properties of $x(t)=e^{*}(t) A(t)$ are reflected in smoothness of the function $e(t)$ and multiplicity bounds on the martingale $A(t)$. In this section we discuss the elementary aspects of this theory.

To avoid trivialities we will assume that the martingale $A(t)$ is nonsingular. The basic identity which follows directly from the martingale property of $A(t)$ is

$$
\begin{aligned}
\|x(t)-x(s)\|^{2}= & \left\|e^{*}(t)[A(t)-A(s)]\right\|^{2}+\left\|(e(t)-e(s))^{*} A(s)\right\|^{2} \\
= & e^{*}(t)[G(t)-G(s)] e(t) \\
& +(e(t)-e(s))^{*} G(s)(e(t)-e(s)), \quad s \leq t .
\end{aligned}
$$

From (V.1) we have at once

$$
\|x(t)-x(s)\|^{2} \geq e^{*}(t)[G(t)-G(s)] e(t),
$$

and

$$
\|x(t)-x(s)\|^{2} \geq \lambda(s)|e(t)-e(s)|^{2}, \quad s \leq t
$$

where $\lambda(s)$ is the minimum eigenvalue of $G(s)$. The function $\lambda(s)$ is nondecreasing. Because $G(s)$ is non-singular $\lambda(s)>0$.

From (V.3) it is clear that if $x(t)$ is continuous then so is $e(t)$, and if $x(t)$ satisfies a local Hölder condition then so does $e(t)$. We will now see that differentiability properties are similar. Suppose that

$$
D_{-} x(t)=\lim _{s \uparrow t} \frac{x(t)-x(s)}{t-s}
$$

exists. Upon writing

$$
\frac{x(t)-x(s)}{t-s}=e^{*}(t) \frac{[A(t)-A(s)]}{t-s}+\frac{(e(t)-e(s))^{*}}{t-s} A(s)
$$

and noting by (V.3) that $(t-s)^{-1}|e(t)-e(s)|$ is bounded, we may choose 
a sequence $s_{n} \uparrow t$ so that $\left(t-s_{n}\right)^{-1}\left(e(t)-e\left(s_{n}\right)\right)^{*} A\left(s_{n}\right)$ converges as $n \rightarrow \infty$. Thus $e^{*}(t)\left(t-s_{n}\right)^{-1}\left[A(t)-A\left(s_{n}\right)\right]$ also converges to some limit, say $x$. But by the martingale property, $x$ is orthogonal to $\operatorname{sp}\{x(s): s<t\}$. On the other hand, since $x(s)$ is left continuous at $t, \operatorname{sp}\{x(s): s<t\}=\mathscr{H}(x, t)$ and $x$ is orthogonal to

$$
x=\left[D_{-} x(t)-\lim _{n \rightarrow \infty} \frac{\left[e(t)-e\left(s_{n}\right)\right]^{*}}{t-s_{n}} A\left(s_{n}\right)\right] \in \mathscr{H}(t, x) .
$$

Thus $x=0$ and

$$
D_{-} x(t)=\lim _{n \rightarrow \infty} \frac{\left[e(t)-e\left(s_{n}\right)\right]^{*}}{t-s_{n}} A\left(s_{n}\right)
$$

As this must be true for any convergent subsequence we must have

$$
D_{-} x(t)=\lim _{s \uparrow t} \frac{[e(t)-e(s)]^{*}}{t-s} A(s) .
$$

This and the assumption that $A(t)$ is non-singular now imply that $D_{-} e(t)$ exists and $D_{-} x(t)=\left(D_{-} e(t)\right)^{*} A(t-)$. Similarly, if $x(t)$ is $k$ times differentiable then so is $e(t)$ and

$$
x^{(k)}(t)=\left(e^{(k)}(t)\right)^{*} A(t)
$$

THEOREM V.1. Suppose $x(t)$ is $(k-1)$ times differentiable and that for each $t,\left\{x(t), \cdots, x^{(k-1)}(t)\right\}$ are linearly independent. Then:

(i) If $x^{(k-1)}(t)$ is continuous the discrete multiplicity of $A(t)$ is bounded by $n-k$.

(ii) If $x^{(k-1)}(t)$ is Hölder $\alpha$-continuous for some $\alpha>1 / 2$ then the multiplicity of $A(t)$ is bounded by $n-k$.

Proof. (i) For each $t$ the $k$ vectors $e(t), \cdots, e^{(k-1)}(t)$ are independent, and applying (V.2) to the derivatives $x^{(j)}(t)$, which we may by (V.4), we have

$0=\left\|x^{(j)}(t+)-x^{(j)}(t-)\right\|=\left(e^{(j)}(t)\right)^{*}(G(t+)-G(t-)) e^{(j)}(t), \quad 0 \leq j<k$.

Thus rank $(G(t+)-G(t-))=n-\operatorname{dim} \operatorname{ker}(G(t+)-G(t-)) \leq n-k$.

(ii) For any interval $[a, b] \subset(0, \infty)$ and any $j=0, \cdots, k-1$ there exists a $c<\infty$ for which

$$
\left\|x^{(j)}(t)-x^{(j)}(s)\right\| \leq c|t-s|^{\alpha}, \quad s, t \in[a, b] .
$$


We now approximate the integral

$$
\int_{a}^{b}\left(e^{(j)}(t)\right)^{*} G^{\prime}(t) e^{(j)}(t) d \mu=\int_{a}^{b}\left(e^{(j)}(t)\right)^{*} G(d t) e^{(j)}(t)
$$

by Riemann-Stieltjes sums which we estimate using (V.2) and (V.5)

$$
\begin{aligned}
\sum\left(e^{(j)}\left(t_{i}\right)\right) *\left[G\left(t_{i}\right)-G\left(t_{i-1}\right)\right] e^{(j)}\left(t_{i}\right) & \leq \sum\left\|x^{(j)}\left(t_{i}\right)-x^{(j)}\left(t_{i-1}\right)\right\|^{2} \\
& \leq c \sum\left|t_{i}-t_{i+1}\right|^{2 \alpha} .
\end{aligned}
$$

Since $2 \alpha>1$ this estimate tends to 0 as the norm of the partition tends to zero. Thus

$$
\int_{a}^{b}\left(e^{(j)}(t)\right)^{*} G^{\prime}(t) e^{(j)}(t) d \mu=0
$$

and since the interval $[a, b]$ was arbitrary we see the kernel of $G^{\prime}(t)$ contains the $k$ independent vectors $\left\{e(t), \cdots, e^{(k-1)}(t)\right\}$ a.s. [ $\left.\mu\right]$ and $\operatorname{rank} G^{\prime}(t) \leq n-k$.

COROLlaRY V.2. If $k=n-2$ in Theorem V.1 part (ii) then $x(t)$ has simple spectrum.

\section{REFERENCES}

[1] Cramér, H. On the structure of purely non-determinist stochastic processes, Ark. Mat. 4 (1961), 249-266.

[2 ] Dunford, N. and Schwartz, J. Linear Operators, II, Interscience, New York (1963).

[ 3 ] Hida, T. Canonical representations of Gaussian processes and their applications, Mem. Coll. Sci. Univ. Kyoto, Ser. A, 33 (1960), 109-155.

[4] Hitsuda, M. Multiplicity of some classes of Gaussian processes, Nagoya Math. J. 52 (1973), 39-46.

[5] Lévy, P. Wiener's random function and other Laplacian random functions, Proc. 2nd Berkeley Symp. Math. Stat. and Prob., Univ. Calif. Press, Berkeley (1951), 171-187.

[6] Lévy, P. A special problem of Brownian motion and a general theory of Gaussian random functions, Proc. 3rd. Berkeley Symp. Math. Stat. and Prob. II, Univ. Calif. Press, Berkeley (1956a), 133-175.

[ 7 ] Lévy, P. Sur une classe de courbes de l'espace Hilbert et sur une equation integrale non lineaire, Ann Ecole Norm. Sup 73 (1956b), 121-156.

[ 8 ] Mandrekar, V. On multivariate wide-sense Markov processes, Nagoya Math. J. 33 (1968), 7-19.

[9] Mandrekar, V. On the multiple Markov property of Lévy-Hida for Gaussian processes, Nagoya Math. J. 54 (1974), 69-78.

[10] Rosenberg, M. Square integrability of matrix valued functions with respect to a non-negative definite Hermitian measure, Duke Math. J. 31 (1964), 291-298. 
[11] Rozanov, Yu. Spectral theory of multidimensional stationary random processes with discrete time, Uspehi Mat. Nauk (N.S.) 13 No. 2 (80) (1958), 93-142 Selected. transl. in Math. Stat. and Prob. I (1961), 127-132.

University of Virginia 